\title{
Chaotic transport in low-dimensional superlattices
}

\author{
Michael Zwolak, David Ferguson, and Massimiliano Di Ventra* \\ Department of Physics, Virginia Polytechnic Institute and State University, Blacksburg, Virginia 24061-0435
}

(Received 18 November 2002; published 14 February 2003)

\begin{abstract}
We predict that in arrays of quantum dots (OD superlattice) and arrays of one-dimensional quantum wires (1D superlattice) chaotic transport should be observed in the presence of an ac field and for a wide range of physical parameters, like the external dc bias, contact charge, doping levels, and disorder in the array. Timedependent current oscillations set in the array due to the formation of electric domain walls when sequential resonant tunneling is the main transport mechanism between adjacent units. Such oscillations can then be forced into spatiotemporal chaos. A similar phenomenon has been predicted and demonstrated for solid-state superlattices. However, contrary to the latter case, the domain walls move across a larger number of units in the superlattice the lower the dimensionality, due to the different spatial distribution of the electric-field across the array in the three cases.
\end{abstract}

DOI: 10.1103/PhysRevB.67.081303

Chaotic transport in GaAs/AlAs superlattices has been theoretically predicted ${ }^{1}$ and experimentally demonstrated ${ }^{2}$ a few years ago. It was found that, when the main charge transport mechanism is sequential resonant tunneling between adjacent quantum wells, undamped time-dependent oscillations of the current appear. ${ }^{3}$ Such oscillations are due to the motion and recycling of electric field and charge domain walls in the superlattice. ${ }^{1}$ Due to the presence of such natural oscillations, spatiotemporal chaos can be induced by a suitable external oscillating field. ${ }^{1,4}$

In recent years, new type of nanostructures, like, e.g., nanotubes, molecular or atomic wires (often globally referred to as quantum wires), have received considerable attention in view of their possible use as components in future electronic applications. ${ }^{5}$ While much of the research has focused so far on the new transport issues that arise in single quantum wires, less work has been devoted to the study of transport properties of arrays of quantum wires. The latter systems constitute a natural step towards integration of nanoscale components into functional devices. Since such systems represent an extension of the well-known concept of two-dimensional (2D) solid-state superlattices ${ }^{6}$ and sequential resonant tunneling can be the main transport mechanism in such structures, it is natural to ask if (i) natural oscillations can be observed in superlattices with even lower dimansionality, and, consequently, (ii) such oscillations can be forced into spatiotemporal chaos by an appropriate oscillating field.

In this paper, we show that the answer to both questions is positive. In particular, we examine arrays of quantum dots (OD superlattice) and arrays of one-dimensional quantum wires (1D superlattice). By array of quantum wires we mean either a finite series of zero dimensional (0D) structures [see Fig. 1(a)] or a series of one dimensional (1D) structures [Fig. 1(b)] between two bulk electrodes. The 0D superlattice can be made of, for instance, a series of weakly coupled molecules, ${ }^{7}$ or a series of nanowire units, ${ }^{8}$ and the 1D superlattice a series of, say, nanotubes. ${ }^{5}$ We consider both homogeneous arrays and inhomogeneous arrays (i.e., arrays with aperiodic lattice spacing or with variations in electronic structure from one unit of the array to the next. ${ }^{9}$ ) In homogeneous arrays, electric domain walls form, leading to time-
PACS number(s): 73.63.Nm, 05.45.Ac, 85.35.-p dependent current oscillations as in the case of 2D superlattices. However, contrary to the $2 \mathrm{D}$ case, the domain walls move across a larger number of units in the array the lower the dimensionality. The difference is due to the different spatial distribution of the electric-field across the array in the three cases. Spatiotemporal chaos can then be induced by an ac field and for a wide range of physical parameters, like the external bias, contact charge, and doping levels. Inhomogeneous arrays display qualitatively similiar behavior. Due to the wide range of parameters and insensitivity to disorder, our predictions should be readily tested experimentally.

Model. We consider as in Ref. 1 an array of $N$ elements (from now on called unit cells) with lattice spacing $L$ (see Fig. 1). In this case, however, each unit cell can host either a point-charge (0D case) or a line charge density (1D case). We also assume that the main charge transport mechanism is provided by sequential resonant tunneling. This assumption

(a)

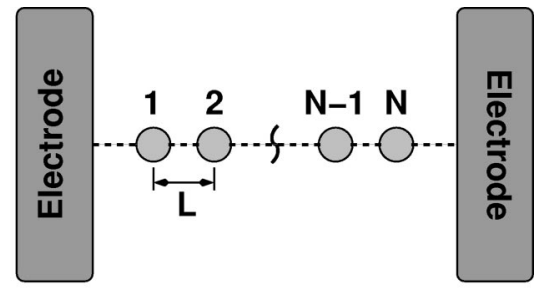

(b)

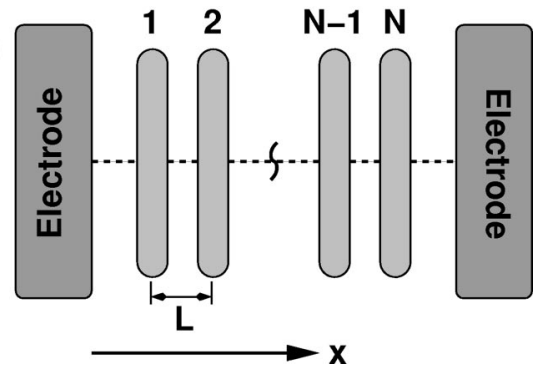

FIG. 1. Schematic of the two sytems under study. The 0D (a) and 1D (b) superlattices are arrays of $N$ point elements and line elements, respectively. There is an excess charge $n_{j}(j=1, \ldots, N)$ and doping charge $n_{D}$ on each object. The array has a contact charge $\delta$ and lattice spacing $L$. The right electrode is held at potential $V$ compared to the left electrode. 
allows us to treat the charge transport semiclassically. ${ }^{1}$ For each unit cell, there is an excess charge $n_{j}(j=1, \ldots, N)$ and a doping charge $n_{D}$. In the 0D case, $n_{j}$ and $n_{D}$ are the excess charge and doping charge, respectively. In the 1D case, $n_{j}$ and $n_{D}$ are the excess line charge density and line charge doping density. We keep the right contact at a dc bias $V$ compared to the left contact. We also assume that there is a contact charge $\delta$ between the first unit cell and the left electrode. The value of such charge can vary from a considerable small fraction of the doping charge in the GaAs/AlAs superlattice case ${ }^{1}$ to a fraction of an electron in the case of molecules in contact with gold electrodes. ${ }^{7}$ The main quantity that affects the charge transport is the electrostatic potential $\Phi(x)$ along the transport direction. The latter is a linear function of the excess charge in the unit cells, their image charges, and the charge at the contacts. The electrostatic potential can be written as

$$
\Phi(x)=\Phi^{\circ}(x)+\sum_{j=1}^{N} \frac{\partial \Phi(x)}{\partial n_{j}} n_{j} .
$$

Here,

$$
\Phi^{\circ}(x)=\frac{-\sigma x}{\epsilon}=\frac{V x}{(N+1) L},
$$

where $\sigma=\sigma_{\text {Left }}{ }^{\circ}=-\sigma_{\text {Right }}{ }^{\circ}$ is the surface charge on the electrodes, and we assume that the unit cells in the superlattice are embedded in a dielectric material of permittivity $\epsilon$. The derivatives $[\partial \Phi(x)] / \partial n_{j}$ are due to the excess charge and their image charges. Since the excess charges and image charges are symmetric about each contact the following boundary conditions are satisfied (see Fig. 1):

$$
\left.\frac{\partial \Phi(x)}{\partial n_{j}}\right|_{N+1}=\left.\frac{\partial \Phi(x)}{\partial n_{j}}\right|_{0}=0 .
$$

We can rewrite Eq. (2) in a compact form, valid in all dimensions, by introducing a vector $\overrightarrow{\Delta \Phi}$ whose $i$ th element is the potential difference between the $(i+1)$ th and the $i$ th unit cell in the array

$$
\overrightarrow{\Delta \Phi}=\mathbf{A} \vec{n}+\overrightarrow{1} \frac{V}{N+1} .
$$

$\mathbf{A}$ is a matrix whose elements are

$$
a_{i j}=\left.\frac{\partial \Phi(x)}{\partial n_{j}}\right|_{i+1}-\left.\frac{\partial \Phi(x)}{\partial n_{j}}\right|_{i}
$$

and $\overrightarrow{1}$ is a vector with all elements equal to one. For the different dimensionalities of the unit cells (0D, 1D, or 2D), $\mathbf{A}$ takes on different forms. ${ }^{10}$ Aperiodic lattice spacing affects $\mathbf{A}$ through the distance between unit cells and how the bias $V$ is divided among the unit cells.

For simplicity, we define dimensionless units as follows: The dimensionless potential drop is $\Delta \phi=\Delta \Phi / \Delta \Phi_{\text {peak }}$, where $\Delta \Phi_{\text {peak }}$ is the potential difference between any two unit cells that induces resonant tunneling between them, while the dimensionless velocity is $\bar{v}(\Delta \phi)=v(\Delta \Phi)$ /
$v\left(\Delta \Phi_{\text {peak }}\right) \cdot{ }^{11}$ Its form is assumed to be the same as in the $2 \mathrm{D}$ case (see also discussion below). ${ }^{1,12}$ We define $t_{\text {tun }}$ $=L / v\left(\Delta \Phi_{\text {peak }}\right)$ and the dimensionless time as $\tau=t / t_{\text {tun }}$. We define $\mathcal{V}=V / \Delta \Phi_{\text {peak }}(N+1)$ as the dc bias amplitude between two unit cells. The dimensionless excess charge density is

$$
\bar{n}_{i}=\left\{\begin{array}{l}
n_{i} / 4 \pi \epsilon L \Delta \Phi_{\text {peak }} \rightarrow 0 \mathrm{D} \\
n_{i} / 2 \pi \epsilon \Delta \Phi_{\text {peak }} \rightarrow 1 \mathrm{D} .
\end{array}\right.
$$

The dimensionless doping density, $\bar{n}_{D}$ is defined similarly. We later include an ac bias of the form

$$
V_{c}=V A_{c} \sin (\omega t),
$$

where $A_{c}$ is the relative magnitude of the ac bias to the $\mathrm{dc}$ bias. We can then define $a=A_{c} \mathcal{V}$ and the dimensionless driving frequency $\bar{\omega}=\omega t_{t u n}$.

Since we are assuming sequential resonant tunneling, we can evaluate the change in excess charge on each unit cell $i$ as

$$
\frac{d \bar{n}_{i}}{d \tau}=\left(\bar{n}_{i-1}+\bar{n}_{D}\right) \bar{v}\left(\Delta \phi_{i-1}\right)-\left(\bar{n}_{i}+\bar{n}_{D}\right) \bar{v}\left(\Delta \phi_{i}\right)
$$

with boundary conditions

$$
\frac{d \bar{n}_{1}}{d \tau}=0, \quad \bar{n}_{1}=\delta .
$$

Finally, the dimensionless current-density $J$ is the sum of the electric current and the displacement current,

$$
J=\left(\bar{n}_{N}+\bar{n}_{D}\right) \Omega \bar{v}\left(\Delta \phi_{N}\right)-\frac{\partial \Delta \phi_{N}}{\partial \tau} .
$$

For the $0 \mathrm{D}$ case, $\Omega=4 \pi L^{2} / A$, where $A$ is the area of the unit cell perpendicular to the current, and we take $A$ as $\pi(L / 4)^{2}$. For the 1D case, $\Omega=2 \pi l L / A$, where $l$ is the length of the unit cell, and we take $A$ as $l L / 2$.

In the presence of the ac bias, the total current-density $J$ can be written as

$$
J=\left(\bar{n}_{i}+\bar{n}_{D}\right) \Omega \bar{v}\left(\Delta \phi_{i}\right)-\sum_{j=1}^{N} \bar{a}_{N, j} \frac{d \bar{n}_{j}}{d \tau}-a \bar{\omega} \cos \bar{\omega} \tau,
$$

where $\bar{a}_{N, j}$ are the dimensionless matrix elements [from Eq. (5)]. ${ }^{13}$ In the case of inhomogeneous arrays, Eqs. (8), (10), and (11) are slightly modified due to different distances between unit cells and/or different energy-level separation from cell to cell.

Current oscillations. Let us first study a homogeneous array with zero ac field. As an example, we consider 40 unit cells with dimensionless contact charge of $\sim 0.05$ and dimensionless doping charge of 0.05 , for all three dimensionalities. ${ }^{14} \mathrm{We}$ will discuss later the range of parameters for which current oscillations and chaos can be observed. We find that, as in the $2 \mathrm{D}$ case, current oscillations and domain walls form for both the $0 \mathrm{D}$ and $1 \mathrm{D}$ cases, as 
(a)

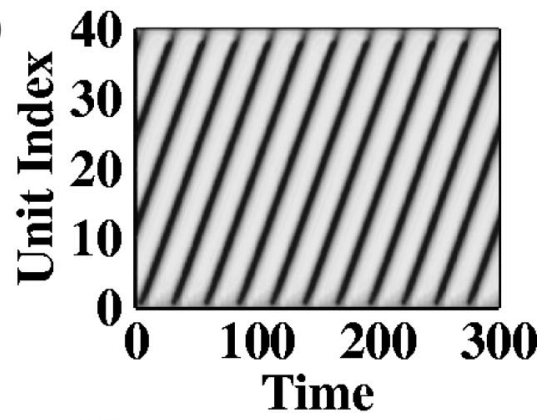

(b)

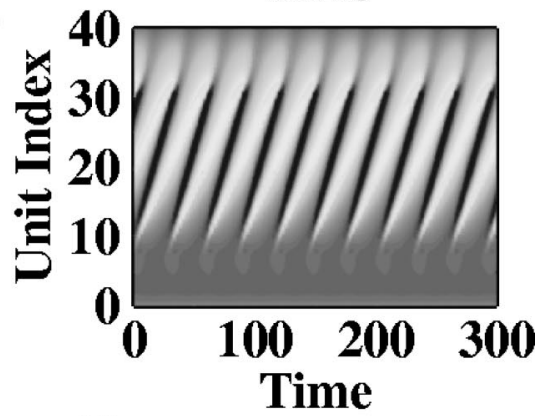

(c)

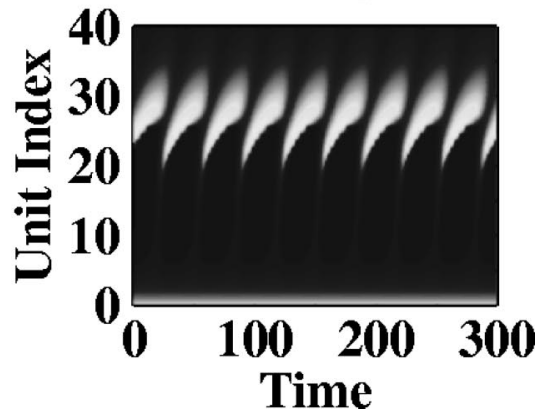

FIG. 2. Charge density accross the OD (a) and 1D (b) homogeneous superlattices as a function of (dimensionless) time. The charge density for the 2D superlattice (c) is also shown for comparison. Light regions indicate charge accumulation.

shown in Fig. 2, where the charge-density along the superlattice is plotted as a function of time for the three cases. For this particular set of parameters and $t_{\text {tun }}=2 \mathrm{~ns}$, the frequency of the oscillations is $\sim 15 \mathrm{MHz}$ for the $2 \mathrm{D}$ case, $\sim 17 \mathrm{MHz}$ for the $1 \mathrm{D}$ case and $\sim 18 \mathrm{MHz}$ for the $0 \mathrm{D}$ case. The domain walls are formed inside the superlattice and move towards the opposite contact (Fig. 2). Depending on the external bias, however, they can dissolve before reaching the contact. ${ }^{1}$ It is also evident from Fig. 2 that the domain walls move across a larger number of unit cells the lower the dimensionality. Consequently, several domain walls can coexist in the superlattice at a given time, and their number increases with decreased dimensionality. The trend can be easily understood in terms of the differences in the way the electric field from the excess charge decays across the unit cells. In the 0D case, the electric-field drops off as $1 / x^{2}$; thus, the excess charge has little long-range effect. In this case, at any given time, a domain wall already present in the array has little effect in blocking the formation of a new domain at the contact at the same time. Note that, incidentally, the single charge domains in this case are also distributed on a larger number of unit cells than in the 1D and 2D cases (see Fig. 2). In the 1D case, the electric field decays slower (as $1 / x$ ). This causes the

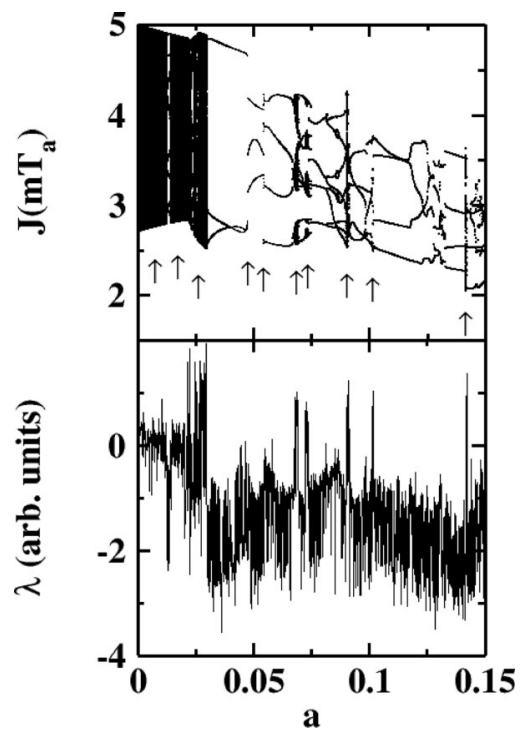

FIG. 3. Poincaré map (upper panel) and Lyapunov exponents (lower panel) for the homogeneous 0D case. The plot shows current at time intervals equal to $m T_{a}$ (where $m$ is an integer) versus the relative ac field bias $a$. Arrows indicate the chaotic regions.

domain walls to be more localized in the middle of the superlattice with fewer present at any one time. This effect is even increased in the $2 \mathrm{D}$ case, where the electric field is constant.

Chotic transport. Having found these current oscillation we can now force them with an additional ac bias. As in the 2D case, ${ }^{1}$ we fix the ratio between the natural frequency and the driving frequency to be the golden mean $(\sqrt{5}-1) / 2$. We then find chaotic transport for selected values of the relative field strength $a$ for both the $0 \mathrm{D}$ and 1D cases. Figures 3 and 4 (upper panels) show the Poincare map of the current for 0D and 1D, respectively, as a function of $a$. The current is plot-

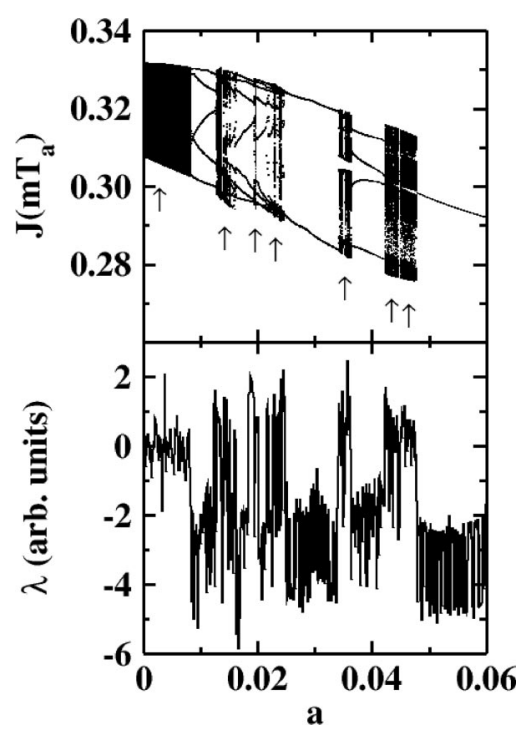

FIG. 4. Poincaré map (upper panel) and Lyapunov exponents (lower panel) for the homogeneous 1D case. The plot shows current at time intervals equal to $m T_{a}$ (where $m$ is an integer) versus the relative ac field bias $a$. Arrows indicate the chaotic regions. 
ted at intervals of the applied ac bias period, i.e., at intervals $m T_{a}$, where $m$ is an integer and $T_{a}$ is the ac bias period. We find several regions of chaos in both cases, as indicated by the arrows that correspond to positive Lyapunov exponents (lower panel in Figs. 3 and 4). We have also found that in the regions of chaotic transport there is loss of spatial coherence due to chaotic domain-wall dynamics as in the $2 \mathrm{D}$ case. ${ }^{1}$

We conclude this paper by discussing the range of parameters to obtain chaotic transport in 0D and 1D superlattices and the effects of inhomogeneities in the superlattice. If we fix the lattice spacing $L$, to be $1 \mathrm{~nm}$, the permittivity of the background medium $\epsilon=10 \epsilon_{0}$, and no doping charge, we find chaotic transport for external electric fields of about $10^{5} \mathrm{~V} / \mathrm{cm}$, and contact charges of $\sim 0.01 e$ for the $0 \mathrm{D}$ case and $\sim 0.002 e / \AA$ for the $1 \mathrm{D}$ case. Both the electric-field range and contact charges are quite reasonable for nanoscale devices. ${ }^{7}$ Within the same range of parameters we find formation of domain walls and chaos for arrays with a number of unit cells as small as four for $0 \mathrm{D}$, and eight for 1D. Reducing the lattice spacing or electric-field necessary for resonance reduces the contact charge necessary for the formation of domain walls. The velocity profile has a sharper shape in the $1 \mathrm{D}$ and $\mathrm{OD}$ cases due to the different density of states compared to to the $2 \mathrm{D}$ case. ${ }^{15}$ We find that sharper velocity profiles favor chaotic behavior.
Given a set of physical parameters that produce chaotic transport, spatial disorder with gaussian distribution of width as small as $\sim 0.02 L$ can destroy chaos. However, a change in device parameters, like, e.g., reducing the contact charge, can reintroduce both current oscillations and chaos for spatial disorder of up to $\sim 0.1 L$ for both broad and sharp velocity profiles. Finally, random variation of the energy levels of each unit cell up to $\sim 0.2 \Delta \Phi_{\text {peak }}$ has essentially no effect on the chaotic behavior of the superlattice.

In conclusion, we predict that spatiotemporal chaos should be observed in arrays of weakly coupled quantum wires under appropriate external dc and ac biases when sequential resonant tunneling is the main charge transport mechanism. We also find that the domain walls move across a larger number of units in the array the lower the dimensionality. Chaotic regions should be observed for both homogeneous and inhomogeneous arrays for a wide range of physical parameters, like the external dc bias, contact charge, and doping levels. Such a wide range of parameters should permit an experimental confirmation of our predictions.

We acknowledge support from the NSF Grants Nos. DMR-01-02277 and DMR-01-33075, and Carilion Biomedical Institute. Acknowledgement is also made to the Donors of The Petroleum Research Fund, administered by the American Chemical Society, for partial support of this research.
*Corresponding author. Email address: diventra@vt.edu

${ }^{1}$ O.M. Bulashenko and L.L. Bonilla, Phys. Rev. B 52, 7849 (1995); O.M. Bulashenko, M.J. Garcia, and L.L. Bonilla, ibid. 53, 10008 (1996). For a recent review, see, e.g., A. Wacker, Phys. Rep. 357, 1 (2002).

${ }^{2}$ Y. Zhang, J. Kastrup, R. Klann, K.H. Ploog, and H.T. Grahn, Phys. Rev. Lett. 77, 3001 (1996).

${ }^{3}$ Note that domain walls have been predicted also in wideminiband superlattices due to the negative effective mass of Bloch electrons in the miniband [see, e.g., J.C. Cao, H.C. Liu, X.L. Lei, and A.G. U Perera, Phys. Rev. B 63, 115308 (2001), and references therein], and also under time-independent external voltage conditions [see, e.g., A. Amann, J. Schlesner, A. Wacker, E. Schöll, ibid. 65, 193313 (2002)]. We leave these cases for future studies

${ }^{4}$ K.J. Luo, H.T. Grahn, K.H. Ploog, and L.L. Bonilla, Phys. Rev. Lett. 81, 1290 (1998).

${ }^{5}$ See, e.g., M.S. Dresselhaus, G. Dresselhaus, and P.C. Eklund, Science of Fullerenes and Carbon Nanotubes (Academic, San Diego, CA, 1996); Molecular Electronics II, edited by A. Aviram, M.A. Ratner and V. Mujica (New York Academy of Sciences, New York, 2002).
${ }^{6}$ L. Esaki and R. Tsu, IBM J. Res. Dev. 14, 61 (1970).

${ }^{7}$ M. Di Ventra and N.D. Lang, Phys. Rev. B 65, 045402 (2002); M. Di Ventra, S.T. Pantelides, and N.D. Lang, Phys. Rev. Lett. 84, 979 (2000); M. Di Ventra, S.T. Pantelides, and N.D. Lang, Appl. Phys. Lett. 76, 3448 (2000).

${ }^{8}$ M.S. Gudiksen, L.J. Lauhon, J. Wang, D.C. Smith, and C.M. Lieber, Nature (London) 415, 617 (2002).

${ }^{9}$ Variations in electronic structure can be caused, for instance, by variations in unit cell size and shape.

${ }^{10}$ M. Zwolak, D. Ferguson, and M. Di Ventra (unpublished).

${ }^{11}$ We will consider in the following a value of the external dc bias that induces negative differential resistance across the unit cells.

${ }^{12}$ L.L. Bonilla, G. Platero, and D. Sanchez, Phys. Rev. B 62, 2786 (2000); R. Aguado, G. Platero, M. Moscoso, and L.L. Bonilla, ibid. 55, 16053 (1997).

${ }^{13}$ Note that this expression of the current reduces exactly to that reported by Bulashenko and Bonilla for the 2D case (See Ref. 1).

${ }^{14}$ We want to stress that in the $0 \mathrm{D}$ and $1 \mathrm{D}$ case current oscillations and chaos can also be obtained with no doping.

${ }^{15}$ See, e.g., M. Narihiro, G. Yusa, Y. Nakamura, T. Noda, and H. Sakaki, Appl. Phys. Lett. 70, 105 (1997). 